\title{
Preliminary investigation of a biological augmentation of rotator cuff repairs using a collagen implant: a 2-year MRI follow-up
}

\author{
Desmond John Bokor ${ }^{1}$ \\ David Sonnabend ${ }^{2}$ \\ Luke Deady ${ }^{3}$ \\ Ben Cass ${ }^{4}$ \\ Allan Young 4 \\ Craig Van Kampen ${ }^{5}$ \\ Steven Arnoczky6
}

1 Department of Orthopaedic Surgery, Faculty of Medicine and Health Sciences, Macquarie University, Sydney, Australia

2 Department of Orthopaedic Surgery, Royal North Shore Hospital, St. Leonards, Australia

3 Alfred Imaging \& Alfred Advanced Sports Imaging Centre, Drummoyne, Australia

4 Sydney Shoulder Specialists, St. Leonards, Australia

5 Rotation Medical, Inc., Plymouth, MN, USA

6 College of Veterinary Medicine, Michigan State University, East Lansing, MI, USA

Corresponding author:

Desmond John Bokor

Department of Orthopaedic Surgery, Faculty of Medicine and Health Sciences, Macquarie University

Technology Place 2

2109 Sydney, Australia

E-mail: desbok@iinet.net.au

\section{Summary}

Background: the inability to restore the normal tendon footprint and limit strains on the repair site are thought to contribute to re-tearing following rotator cuff repair. The purpose of this study was to use a collagen implant to augment rotator cuff repairs through the restoration of the native tendon footprint and the induction of new tissue to decrease overall tendon strain.

Methods: repairs of full-thickness rotator cuff lesions in 9 adult patients were augmented with a novel collagen implant placed over the bursal surface of the repair. Tendon thickness and footprint anatomy were evaluated using MRI at 3,6,12, and 24 months. Clinical results were assessed using standard outcome metrics. Mean follow-up for all patients was 25.8 months.

Results: the implant induced significant new tissue formation in all patients by 3 months. This tissue matured over time and became indistinguishable from the underlying tendon. At 24 months all repairs remained intact and normal footprint anatomy of the tendon was restored in all patients. All clinical scores improved significantly over time.

Conclusion: the ability of a collagen implant to induce new host tissue formation and restore the normal footprint anatomy may represent a significant advancement in the biological augmentation and ultimate durability of rotator cuff repairs.

KEY WORDS: collagen implant, cuff repair, footprint, tendon.

\section{Introduction}

Rotator cuff repair is one of the most common orthopaedic procedures performed, with between 200,000 and 300,000 rotator cuffs being surgically repaired annually ${ }^{1,2}$. Although surgical techniques for rotator cuff repair have advanced over the last decade, one of the most important postoperative complications of rotator cuff repair is re-tear of the repaired tendon ${ }^{3}$. Recent estimates suggest that retear rates in rotator cuff repair are in the order of 11 to $57 \%{ }^{3}$. While studies have suggested that re-torn repairs can still provide clinical benefit to patients, subjective and objective outcome metrics in these patients are inferior to those patients with intact repairs ${ }^{3-5}$.

Re-tear of a rotator cuff repair has been associated with a multitude of factors including patient age, tear dimensions, and tendon tissue quality ${ }^{3}$. However, a recent report of 1000 consecutive rotator cuff repairs found that no single preoperative or intra-operative factor was overwhelmingly predictive of re-tearing ${ }^{3}$. Several studies have suggested that the biomechanical environment of the healing tendon may also play a significant role in the ability to achieve a durable rotator cuff repair6-10. These studies have advocated restoring the normal footprint dimensions of the rotator cuff tendon attachment to bone and decreasing tissue strain in the healing tendon as techniques to improve the long-term integrity of rotator cuff repairs ${ }^{6-10}$. Biomechanical analysis of surgical techniques such as the 'double-row' fixation, which utilizes two rows of tendon fixation to bone to increase the contact area of the tendon attachment to bone, has shown improved initial tendon contact and strength $^{10}$ and also decreased tendon strain ${ }^{8}$. How- 
ever, clinical studies have not shown a significant difference in the re-tear rate between double-row and single-row repairs ${ }^{11-15}$. This would suggest a more biologic solution may be needed to optimize rotator cuff repair durability ${ }^{16}$.

A recent finite element analysis has shown that the creation of $2 \mathrm{~mm}$ of new connective tissue over the bursal surface of the supraspinatus tendon and spanning the normal tendon to bone footprint, would significantly reduce intra-tendinous strain and, thus, could theoretically improve healing and long-term durability of the repaired rotator cuff tendon ${ }^{17}$. In a related preclinical study, the use of a bioinductive implant consisting of highly purified type 1 collagen fibers reconstituted into an aligned and highly porous structure with a low tensile modulus (Rotation Medical, Inc., Plymouth, Minnesota USA) was able to rapidly and consistently induce $2-3 \mathrm{~mm}$ of new tendon-like tissue over the surface of a rotator cuff tendon ${ }^{18}$. In addition, the implant was able to re-establish a biological attachment of this new tissue to bone that was similar to the native direct insertion of the rotator cuff footprint ${ }^{18}$.

Therefore, the purpose of this current clinical study was to evaluate the ability of this collagen implant to improve the integrity and durability of full-thickness rotator cuff tears over a two year period. It was hypothesized that the collagen implant would rapidly induce the formation and functional maturation of new tendon-like tissue over the surface of the full-thickness repairs as determined by sequential magnetic resonance imaging (MRI) over a 24-month period. It was also hypothesized that this new tissue would reestablish the normal rotator cuff footprint, maintain repair integrity, and improve clinical outcomes over the 24-month evaluation period.

\section{Materials and methods}

This study was conducted in accord with international standards and meets the ethical standards of the Muscle, Ligaments, and Tendons Journal ${ }^{19}$. This trial was registered with the Australia New Zealand Clinical Trials Registry (Trial ID: ACTRN12611001082998).

\section{Patient enrollment}

Following Ethics Committee approval, patients with supraspinatus tendon tears that required surgical repair were recruited for a clinical study to determine the effect of a highly-porous collagen implant (Rotation Medical, Inc., Plymouth, Minnesota USA) on tissue induction, footprint restoration, repair integrity, and clinical outcomes over a $24-$ month period. All patients signed informed consent.

Inclusion criteria included patient age between 40-66 years old at time of surgery and chronic shoulder pain of more than 3 months duration, which was resistant to analgesics, anti-inflammatory medication and physical therapy. Exclusion criteria included a recent history of steroid use, insulin-dependent diabetes, heavy smoking, genetic collagen disease, chronic inflammatory disease, and index shoulders with previous rotator cuff surgery. Patients with shoulder instability, grade 3 or greater chondromalacia, or grade 2 or greater fatty infiltration of the supraspinatus muscle were also excluded. Finally, the implant is contraindicated for patients with a known hypersensitivity to bovine collagen.

Nine consecutive patients with tears of the supraspinatus tendon, 8 with full-thickness tears and 1 with a high-grade, bursal-sided, partial-thickness tear (which was converted to a full-thickness tear at surgery), met the criteria and were enrolled in the study. Outcome metrics included magnetic resonance imaging (MRI) assessment of tissue induction (change in tendon thickness), footprint restoration, repair integrity and clinical assessment of shoulder pain and function.

\section{Collagen scaffold}

The reconstituted collagen scaffolds were made from highly-purified, type I collagen from bovine tendons using proprietary methods (Collagen Matrix, Inc., Oakland, New Jersey) ${ }^{18}$. The collagen fibers were processed to create a highly oriented and highly porous (85-90\% porosity) collagen scaffold with a DNA content of less than $50 \mathrm{ng} / \mathrm{mg}^{18}$. The scaffolds were freeze-dried after processing and rehydrated at the time of surgery ${ }^{18}$. The rehydrated scaffolds were approximately $2 \mathrm{~mm}$ thick ${ }^{18}$.

\section{Surgical technique}

The surgical procedures were performed under general anesthesia in either the lateral decubitus or beach chair position. Following arthroscopic assessment of the glenohumeral joint, a subacromial bursectomy and decompression were performed and the supraspinatus tendon was repaired prior to attachment of the collagen implant on the bursal surface of the tendon. Rotator cuff repairs were performed with a double-row of suture anchors (5 patients), a doublerow of transosseous sutures (3 patients), or a singlerow of suture anchors (1 patient). If indicated, a biceps tenodesis or tenotomy was also performed.

The collagen implant was placed on the bursal surface of the repaired supraspinatus tendon using custom-designed instrumentation (Rotation Medical, Inc., Plymouth, Minnesota USA). The implant size (20x25 $\mathrm{mm}$ or $25 \times 30 \mathrm{~mm}$ ) was selected to cover almost the entire width of the repaired supraspinatus tendon. Alignment of the implant was centered over the repaired supraspinatus tendon and laterally positioned to overlap onto bone approximately $5 \mathrm{~mm}$ beyond the lateral edge of the supraspinatus footprint. The collagen implant, which is designed to absorb within 6 months, was then affixed to the supraspinatus tendon 
anteriorly, posteriorly, and medially using custom-designed PLA staples, which are made from a PLA copolymer that is designed to absorb in approximately 12 months, and to the bone laterally using customdesigned PEEK staples (Rotation Medical, Inc., Plymouth, Minnesota USA). Care was taken to ensure that the tendon and bone staples did not interfere with the underlying sutures and anchors used in the rotator cuff repair. The implant was attached under a slight amount of tension to assure good contact with the underlying tendon.

\section{Postoperative care}

The use of the collagen implant did not require any changes to the routine postoperative protocol used for patients having a rotator cuff repair. Patients were immobilized in a Velpeau sling during the first 6 weeks, passive motion was allowed, with passive forward flexion limited to $100^{\circ}$ for the first 4 weeks. After 6 weeks the patients progressed as tolerated from active-assisted to active motion under the supervision of an experienced physiotherapist. No resistance exercises were allowed for 12 weeks. After 12 weeks a gradual progressive resistance program was introduced and continued until 26 weeks.

\section{MRI assessment}

Patients underwent MRI evaluation preoperatively and at $3,6,12$, and 24 months postoperatively to assess the integrity of the repair, the relative thickness of the tendon, and the restoration of the tendon attachment footprint.

All of the scans were done at two institutions on 3 Tesla scanners using $2 \mathrm{~mm}$ slices, with proton density (PD) and T2 weighted scans, both with and without fat saturation (fat sat). The preoperative MRI assessment of the rotator cuff tear was confirmed at surgery, where the size of the tear was measured in both the anterior-posterior and medial-lateral directions. All of the MR images were assessed by a single musculoskeletal radiologist who was blinded to the clinical outcomes. These assessments were confirmed by an independent musculoskeletal radiologist who was blinded to both the clinical outcomes and the assessment of the first radiologist. All of the MRI measurements were performed using the PD fat sat images.

In order to most accurately measure the thickness of the supraspinatus tendon over time, the coronal scan was modified to a double-oblique angle aligned with the length of the supraspinatus tendon and perpendicular to its thickness (the oblique coronal plane tilted slightly anteriorly). Thickness measurements were made in the middle (anterior-posterior) of the repair from coronal images at a point just lateral to the articular margin of the supraspinatus insertion (Fig. 1). For each patient, all of the subsequent follow-up thickness measurements were made as close as possible to the location at which the tendon thickness

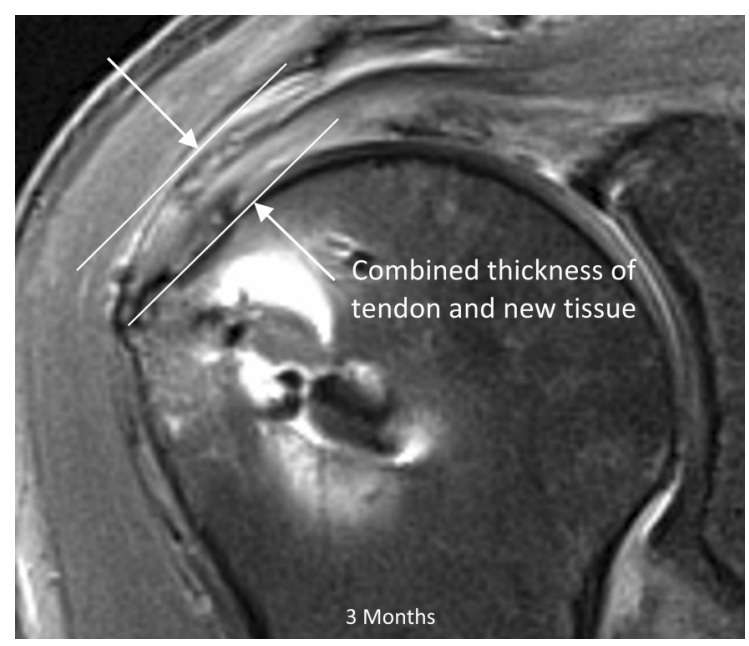

Figure 1. Postoperative MR image at 3 months demonstrating the location (arrows) of the measurement of the combined thickness of the layer of new tissue and the underlying tendon.

was measured in the 3-month MRI. Thickness measurements were compared to previously published ultrasound values for males and females to determine the relative amount of new tissue generation ${ }^{20}$.

Restoration of the footprint area was assessed by evaluation of the MR images at 2 years. The footprint area was considered fully restored if the images indicated that there was tissue in the entire area of the footprint that had the radiological appearance of tendon tissue.

\section{Clinical assessment}

Clinical assessments included use of the ConstantMurley shoulder score (which was not age or gender adjusted) and the American Shoulder and Elbow Society (ASES) shoulder scale, which were both administered preoperatively and at 3, 6, 12, and 24 months postoperatively. Both functional parameters and pain assessment are included in these validated shoulderspecific assessments.

\section{Statistical analysis}

Differences in tendon thickness compared to previously published values for males and females were analyzed using the Student's t-test and changes in clinical scores were assessed using the Friedman two-way ANOVA for non-parametric data. Differences between individual time periods were evaluated using post-hoc analyses and statistical significance was considered at $p<0.05$.

\section{Results}

The 6 male and 3 female patients enrolled in this study had an average age of 56.4 years (range 50-66 
years); 7 right and 2 left shoulders were treated. At time of surgery there were 8 medium-sized full-thickness tears $(1-3 \mathrm{~cm})$ of the supraspinatus tendon and 1 high-grade, bursal-sided, partial-thickness supraspinatus tendon tear $(10 \mathrm{~mm})$ that was converted to a fullthickness lesion at surgery. Five patients were repaired arthroscopically and 4 patients were repaired using a mini-open approach based on the surgeon's preference. In 8 patients, the tendon was reattached using a double-row of suture anchors, while a singlerow repair was used in 1 patient. The mean length of follow-up was 25.8 months (range 24.5-30.4 months), with 24-month follow-up on all 9 patients; no patients were lost to follow-up.

\section{MRI assessment}

MRI evaluation demonstrated no instances of re-tear of the rotator cuff in any of the patents and the integrity of the repaired tendon remained intact in all individuals throughout the 2-year follow-up period.

There was a significant $(p<0.01)$ increase in mean tendon thickness in all subjects at 3 months when compared to published values ${ }^{20}$ (Tab. 1, Fig. 2). While it was possible to delineate newly generated tissue from the native tendon in some patients, other patients had significant edema present in the tendon making it impossible to clearly identify the newly formed tissue. At 6 months all patients continued to demonstrate a significant $(p=0.01)$ increase in tendon thickness (Tab. 1, Fig. 2). This translated into an average of $2 \mathrm{~mm}$ of newly formed tissue over the bursal surface of the supraspinatus tendon. The MRI signal at 6 months revealed that the tendon thickness was still increased and the newly formed tissue was maturing and demonstrated excellent integration of this tissue into the underlying tendon. There was a slight decrease in mean tendon thicknesses from 12 to 24 months, but the values were still significantly ( $p$ $<0.01$ ) greater than that of the published averages ${ }^{20}$. The quality of the new tissue, as well as the underlying tendon, continued to improve and was indistinguishable from the native tendon based on MRI imaging (Fig. 3). This suggests maturation and functional remodeling of the new tissue and a return of the underlying tendon to a more normal state. By 24 months the MRI revealed a continued functional remodeling of the construct with a return towards a normal average thickness values.
Qualitative assessment of the footprint at 24 months by MRI revealed complete coverage of the footprint in all patients (Fig. 4). There was no evidence of any gap formation at any time in any of the patients and all repairs were structurally intact.

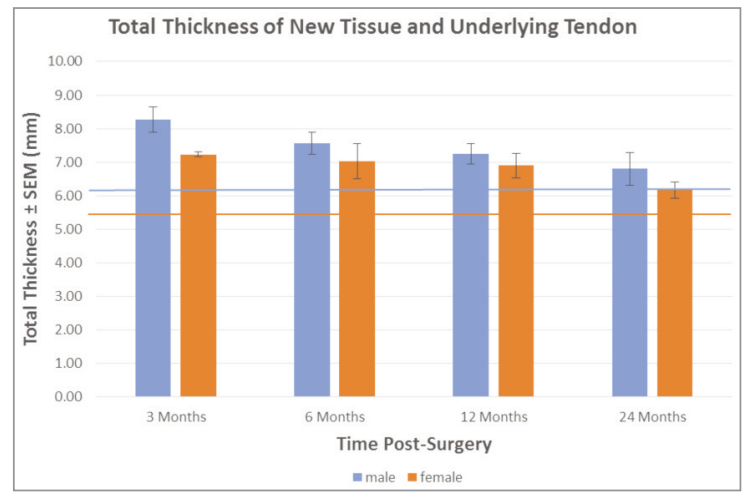

Figure 2. Graph illustrating the overall tendon thickness of male and female patients at each time point compared to published normal average thicknesses (horizontal lines) for males and females ${ }^{20}$.

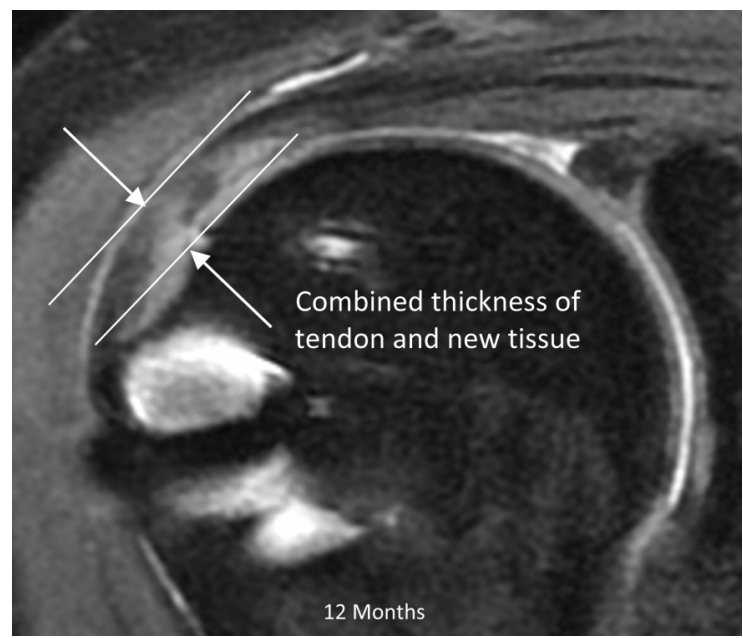

Figure 3. Representative MR image at 12 months illustrating an intact cuff repair. The rotator cuff tendon remains thickened and the newly generated tissue is maturing and is indistinguishable from the underlying tendon. Arrows indicate position where thickness measurement was made. (The white areas in the bone are associated with the bone anchors.)

Table 1. MRI measurement of overall tendon thickness at each time period compared to published normal averages ${ }^{20}$.

\begin{tabular}{|c|c|c|c|c|c|c|c|c|}
\hline & \multicolumn{2}{|l|}{3 Months } & \multicolumn{2}{|l|}{6 Months } & \multicolumn{2}{|c|}{12 Months } & \multicolumn{2}{|c|}{24 Months } \\
\hline & Male & Female & Male & Female & Male & Female & Male & Female \\
\hline $\begin{array}{l}\text { Tendon thickness } \\
\text { Mean } \pm \text { SEM }(\mathrm{mm})\end{array}$ & $8.3 \pm 0.38$ & $7.2 \pm 0.08$ & $7.6 \pm 0.33$ & $7.0 \pm 0.53$ & $7.3 \pm 0.31$ & $6.9 \pm 0.37$ & $6.8 \pm 0.49$ & $6.2 \pm 0.25$ \\
\hline $\begin{array}{l}\text { Published tendon } \\
\text { thickness Mean }(\mathrm{mm})^{20}\end{array}$ & 5.6 & 4.9 & 5.6 & 4.9 & 5.6 & 4.9 & 5.6 & 4.9 \\
\hline Increase in thickness (mm) & 2.7 & 2.3 & 2.0 & 2.1 & 1.7 & 2.0 & 1.2 & 1.3 \\
\hline$p$-value & $<0.01$ & $<0.01$ & $<0.01$ & $<0.01$ & $<0.01$ & $<0.01$ & $<0.01$ & $<0.01$ \\
\hline
\end{tabular}




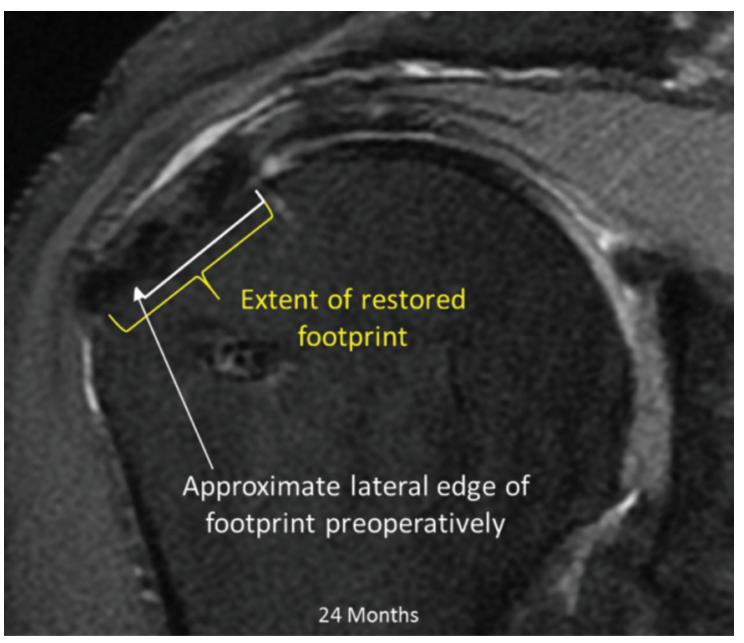

Figure 4. Representative MRI image at 24 months illustrating an intact cuff repair and complete tissue coverage of the restored tendon footprint. The square bracket indicates the approximate location of the footprint preoperatively, with the arrow indicating the lateral edge of the preoperative footprint, suggesting that the area of the footprint has been completely restored. This patient was repaired with transosseous sutures, hence anchors are not evident on the MRI.

\section{Clinical assessment scores}

The overall Constant and ASES scores showed steady improvement throughout the 24 month followup period. At 24 months there were statistically significant improvements over the preoperative scores for both the Constant score $(78.0$ vs $50.7, p<0.001)$ and the ASES score (87.8 vs 44.6, $p<0.001$ ).

Similarly, the Constant and ASES pain scores showed steady improvement throughout the 24 month follow-up period. At 24 months there were statistically significant improvements over the preoperative pain scores for both the Constant (1.1 vs $7.1, p<0.001)$ and ASES (0.7 vs $4.9, p<0.001$ ) scores.

At 24 months $8 / 9(89 \%)$ of the patients had satisfactory results. The patient with the unsatisfactory result did not return for follow-up at 6 and 12 months, but returned at 26 months complaining of pain and intending to file a worker's compensation claim. The 2 year MRI of this patient showed that the repair was intact.

\section{Complications}

One patient in this study, who had pronounced capsulitis preoperatively, also had evidence of capsulitis at 3 months after surgery. While the patient demonstrated steady improvement, there was still some degree of capsular thickening at 24 months. There were no adverse reactions to the collagen implant noted throughout the duration of the study.

\section{Discussion}

A recent systematic review and meta-analysis of rotator cuff literature concluded that despite the increase in the number of articles related to repair of the rotator cuff, the results remain less than ideal with over one-quarter of the repairs in a typical study failing to achieve durable integrity of the rotator cuff at 2 years $^{21}$. While the cause of these re-tears is likely multi-factorial ${ }^{3}$, the inability to restore the native tendon attachment footprint, recreate the normal tendonbone interface (enthesis), and limit excessive strains on the rotator cuff have been implicated as technical reasons for failure to maintain repair integrity ${ }^{7-10,22}$.

The results of the current study have shown that the use of a collagen implant in association with a standard surgical repair of medium-sized $(1-3 \mathrm{~cm})$ rotator cuff tears was able to rapidly induce the generation of new tissue over the bursal surface of the repaired tendon, facilitating the restoration the normal tendon footprint on the humerus, and ultimately maintaining the integrity of the repair for 24 months in all patients. This is in contrast to previous studies which have reported a re-tear rate of up to $29 \%$ in tears of this size $(1-3 \mathrm{~cm})^{3,6}, 23,24$. A recent study has found that re-tears following rotator cuff repair primarily occurred between 6 and 26 weeks, with a substantial number of re-tears occurring between 12 and 26 weeks postoperatively ${ }^{25}$. The ability of the collagen implant used in the current study to rapidly induce a significant amount of new tissue over the rotator cuff repair by 3 months may play a key role in the long-term maintenance of cuff integrity. A finite element analysis has predicted that the addition of a $2 \mathrm{~mm}$ thick layer of connective tissue over the bursal surface of supraspinatus tendon will significantly reduce the intra-tendinous strain ${ }^{17}$. This may, in turn, result in a more optimal biomechanical environment during the time when most re-tears have been reported to occur.

$\mathrm{MRI}$ evaluation of the patients in the current study revealed a significant increase in the generation of new tissue over the bursal surface of the supraspinatus tendon in all patients by 3 months. This increase in tissue thickness persisted over the 24-month evaluation period. During this time, the new tissue demonstrated functional organization, maturation, and remodeling as evidenced by the MRI signal of this new tissue becoming indistinguishable from the native tendon. The slight decrease in overall tendon thickness noted between 12 and 24 months likely reflects the continued functional remodeling of the induced tissue since a previous animal study has demonstrated that the collagen implant is complete gone by 6 months ${ }^{18}$. Additionally, a previous study has demonstrated an association between decreasing tissue stresses and decreasing area of repair tissue in the remodeling of healing tendons ${ }^{26}$. The rapid induction of new tissue, which matured and remodeled into a dense, regularly-oriented connective tissue over time, was similar to the histological progression of the collagen-implant induced tissue formation demonstrated in a preclinical animal study ${ }^{18}$. 
The ability to restore the native footprint and tendonbone interface (enthesis) has been identified as a key factor in optimizing the healing biology in rotator cuff repair $^{9,10,22}$. While results of the current study demonstrate a complete restoration of the footprint by the implant-induced tissue in all patients, the quality of the enthesis can only be surmised by the absence of gap formation at the tendon-bone interface in all patients. However, the aforementioned preclinical animal study has demonstrated the re-creation of a fibrocartilagenous transition zone in the attachment of the implantinduced tissue to bone ${ }^{18}$. The regeneration of a fibrocartilagenous zone at the tendon bone insertion has been suggested to be important for full recovery following tendon to bone repair ${ }^{27}$ and the amount of fibrocartiulage at the tendon-bone insertion is reported to be associated with the strength of the insertion ${ }^{27}$.

All patients in the current study demonstrated a progressive improvement in functional and pain scores over the two year period. These findings are similar to other reported clinical improvements following rotator cuff repair $4,5,23$. While a recent study has suggested that some functional clinical scores are not different between patient with intact repairs and those with retears $^{5}$, other studies have demonstrated a correlation between repair integrity and clinical improvement ${ }^{4,23}$. Additional studies are needed to confirm the importance of cuff integrity on long-term cuff function.

The rationale for the use of extra-cellular matrix (ECM) scaffolds derived from mammalian collagen in rotator cuff repair has previously been based on the ability of the implants to provide initial (time zero) mechanical strength to the repair and/or improve the rate or quality of healing ${ }^{28}$. A recent review of scaffold devices for rotator cuff repair has suggested that the ideal scaffold would serve as an inductive template that would allow for functional remodeling of the repair tissue while limiting or preventing retear of the tendon ${ }^{28}$. While many of the currently available ECM scaffolds have focused on the load sharing characteristics of the scaffold itself ${ }^{28}$, the implant utilized in the current study relies on the rapid induction of host generated connective tissue rather than the initial strength of the scaffold itself to increase tendon mass and thus reduce tendon strain ${ }^{17,} 18$.

There are several potential limitations in this study which must be placed in context. There was no control (no implant) group, the study only examined 9 patients, and the size of the rotator cuff lesions repaired in the current study ranged from $1-3 \mathrm{~cm}$. Although it is well-established that tear size has a direct correlation to re-tear rate $^{3}$, several studies have reported significant re-tear rates of $11 \%$ to $28.6 \%$ in mediumsized $(1-3 \mathrm{~cm})$ tears ${ }^{3}, 6,23,24$. Therefore, using peerreviewed data as historic controls, it could be expected that at least one re-tear would have occurred in a similar number of patients with medium-sized (1-3 $\mathrm{cm}$ ) tears. While the current study provides support for the use of this collagen implant in preventing retears in this population, additional studies are needed to further document the ability of the implant to limit re-tearing in medium, as well as in larger $(>3 \mathrm{~cm})$ ro- tator cuff lesions. In addition, although patients in the current study underwent either arthroscopic or miniopen repair, studies have shown no difference in clinical outcomes or re-tear rates 29,30 . Similar results have also been reported for outcomes and re-tear rates using single- or double-row suture repair techniques ${ }^{15}$. Finally, previously published ultrasound measurements ${ }^{20}$ were used as a reference point on which to base changes in tendon thickness following repair. While these reference ultrasound measurements did not necessarily reflect absolute thickness of the pre-injured tendon, they do provide a fixed standard on which to predicate relative changes in tendon thickness over time.

While additional clinical studies are needed to confirm the role of a biological enhancement technique in maintaining rotator cuff repair integrity, the results of the current preliminary study demonstrate that biological augmentation of rotator cuff repair through the generation of new host tissue represents a novel method which may enhance cuff healing and help to maintain cuff integrity. The ability of a highly-porous, highly-oriented collagen implant to induce new tissue formation and restore the normal tendon footprint represents a significant advancement in the biological augmentation and ultimate durability of rotator cuff repairs.

\section{References}

1. Colvin AC, Egorova N, Harrison AK, Moskowitz A, Flatow EL. National trends in rotator cuff repair. J Bone Joint Surg Am. 2012:94(3):227-233.

2. Ensor KL, Kwon YW, Dibeneditto MR, Zuckerman JD, Rokito AS. The rising incidence of rotator cuff repairs. J Shoulder Elbow Surg. 2013;22(12):1628-1632.

3. Le BT, Wu XL, Lam PH, Murrell GA. Factors predicting rotator cuff retears: an analysis of 1000 consecutive rotator cuff repairs. Am J Sports Med. 2014;42(5):1134-1142.

4. Kim HM, Caldwell JM, Buza JA, Fink LA, Ahmad CS, Bigliani LU, Levine WN. Factors affecting satisfaction and shoulder function in patients with a recurrent rotator cuff tear. J Bone Joint Surg Am. 2014;96(2):106-112.

5. Russell RD, Knight JR, Mulligan E, Khazzam MS. Structural integrity after rotator cuff repair does not correlate with patient function and pain: a meta-analysis. J Bone Joint Surg Am. 2014;96(4):265-271.

6. Ahmad S, Haber M, Bokor DJ. The influence of intraoperatiive factors and postoperative rehabilitation compliance on the integrity of the rotator cuff after arthroscopic repair. J Shoulder Elbow Surg. 2015;24(2):229-235.

7. Lee TQ. Current biomechanical concepts for rotator cuff repair. Clinics Orthop Surg. 2013;5(2):89-97.

8. Kim DH, Elattrache NS, Tibone JE, Jun BJ, DeLaMora SN, Kvitne RS, Lee TQ. Biomechanical comparison of a single-row versus double-row suture anchor technique for rotator cuff repair. Am J Sports Med. 2006;34(3):407-414.

9. Park MC, Tibone JE, EIAttrache NS, Ahmad CS, Jun BJ, Lee TQ. Part II: Biomechanical assessment for a footprint-restoring transosseous-equivalent rotator cuff repair technique compared with a double-row repair technique. J Shoulder Elbow Surg. 2007;16(4):469-476.

10. Wall LB, Keener JD, Brophy RH. Double-row versus singlerow rotator cuff repair: a review of the biomechanical evidence. J Shoulder Elbow Surg. 2009;18(6):933-941. 
11. Burks RT, Crim J, Brown N, Fink B, Greis PE. A prospective randomized clinical trial comparing arthroscopic single- and double-row rotator cuff repair: magnetic resonance imaging and early clinical evaluation. Am J Sports Med. 2009; 37(4):674-682.

12. Franceshi F, Ruzzini L, Longo UG, Martina FM, Zobel BB, Maffulli N, Denaro V. Equivalent clinical results of arthroscopic single-row and double-row suture anchor repair for rotator cuff tears: a randomized controlled trial. Am J Sports Med. 2007; 35(8):1254-1260.

13. Koh KH, Kang KC, Lim TK, Shon MS, Yoo JC. Prospective randomized clinical trial of single-versus double-row suture anchor repair in 2- to 4-cm rotator cuff tears: clinical and magnetic resonance imaging results. Arthroscopy. 2011;27(4):453-462.

14. Ma HL, Chiang ER, Wu HT, Hung SC, Wang ST, Liu CL, Chen TH. Clinical outcome and imaging of arthroscopic single-row and double-row rotator cuff repair: a prospective randomized trial. Arthroscopy. 2012;28(1):16-24.

15. Sheiban-Rad S, Giveans MR, Arnoczky SP, Bedi A. Arthroscopic single-row versus double row rotator cuff repair: a metaanalysis of the randomized clinical trials. Arthroscopy. 2013;29(2):343-348.

16. McCormack RA, Shreve M, Strauss EJ. Biologic augmentation in rotator cuff repair-should we do it, who should get it, and has it worked? Bull Hosp Jt Dis. 2014;72(1):89-96.

17. Chen Q. Two-dimensional finite element proof-of-concept modeling on rotator cuff tear scaffold efficacy. Technical Report from the Material and Structural Testing Core, Mayo Clinic, Rochester, Minnesota, 2011. Retrieved from: http://www. rotationmedical.com/wp-content/uploads/2015/05/fea-whitepaper-4-9-15.pdf.

18. Van Kampen C, Arnoczky S, Parks P, et al. Tissue-engineered augmentation of a rotator cuff tendon using a reconstituted collagen scaffold: a histological evaluation in sheep. Muscles Ligaments Tendons J. 2013;3(3):229-235.

19. Padulo J, Oliva F, Frizziero A, Maffulli N. Muscles, Ligaments and Tendons Journal. Basic principles and recommendations in clinical and field science. MLTJ. 2013;4:250-252.
20. Karthikeyan S, Rai SB, Parsons H, Drew S, Smith CD, Griffin DR. Ultrasound dimensions of the rotator cuff in young healthy adults. J Shoulder Elbow Surg. 2014;23(8):1107-1112.

21. McElvany MD, McGoldrick E, Gee AO, Neradilek MB, Matsen FA 3rd. Rotator cuff repair: published evidence on factors associated with repair integrity and clinical outcome. Am J Sports Med. 2015;43(2):491-500.

22. Lui P, Zhang P, Chan K, Qin L. Biology and augmentation of tendon-bone insertion repair. J Orthop Surg Res. 2010;5:59.

23. Choi S, Kim MK, Kim GM, Roh YH, Hwang IK, Kang H. Factors associated with clinical and structural outcomes after arthroscopic rotator cuff repair with a suture bridge technique in medium, large, and massive tears. J Shoulder Elbow Surg. 2014;23(11):1675-1681.

24. Duquin TR, Buyea C, Bisson LJ. Which method of rotator cuff repair leads to the highest rate of structural healing? A systematic review. Am J Sports Med. 2010;38(4):835-841.

25. Iannotti JP, Deutsch A, Green A, Rudicel S, Christensen J, Marraffino S, Rodeo S. Time to failure after rotator cuff repair: a prospective imaging study. J Bone Joint Surg Am. 2013; 95(11):965-971.

26. Kamps BS, Linder LH, DeCamp CE, Haut RC. The influence of immobilization versus exercise on scar formation in the rabbit patellar tendon after excision of the central third. Am J Sports Med. 1994;22(6):803-811.

27. Chow DHK, Suen PK, Huang L, Cheung W-H, Leung KS, Ng C, Shi S-Q, Wong MWN, Qin L. Extracorporeal shockave enhanced regeneration of fibrocartilage in a delayed tendonbone insertion repair model. J Orthop Res. 2014;32:507-514.

28. Ricchetti ET, Aurora A, lannotti JP, Derwin KA. Scaffold devices for rotator cuff repair. J Shoulder Elbow Surg. 2012;21: 251-265.

29. Shan L, Fu D, Chen K, Cai Z, Li G. All-arthroscopic versus mini-open repair of small to large sized rotator cuff tears: a metaanalysis of clinical outcomes. PLoS One. 2014;9(4):e94421.

30. Ji X, Bi C, Wang F, Wang Q. Arthroscopic versus mini-open rotator cuff repair: an up-to-date meta-analysis of randomized controlled trials. Arthroscopy. 2015;31(1):118-124. 